\title{
Extrapolating contaminant effects from individuals to populations: a case study on nanoparticle toxicity to Daphnia
}

Louise Stevenson ( $\sim$ stevensonlm@ornl.gov )

Oak Ridge National Laboratory https://orcid.org/0000-0003-4967-9897

Katherine Krattenmaker

University of California Santa Barbara

Edward McCauley

University of Calgary

Roger Nisbet

University of California Santa Barbara

Research Article

Keywords:

Posted Date: February 4th, 2022

DOI: https://doi.org/10.21203/rs.3.rs-1312706/v1

License: (c) (i) This work is licensed under a Creative Commons Attribution 4.0 International License.

Read Full License 
January 30,2022

Dear Drs. Desforges, Hickie, Gergs, and Weijs,

Please find the attached manuscript titled "Extrapolating contaminant effects from individuals to populations: a case study on nanoparticle toxicity to Daphnia" for consideration of publication in the special issue in Archives of Environmental Contamination and Toxicology titled "Development and Application of Bioaccumulation and Effect Models to Address Current and Emerging Issues in Ecotoxicology". This is an original manuscript that has not been previously submitted to this or any other journal.

The effective management of ecological risk relies on our ability to predict the effect of stressors on the sustainability of ecosystems. However, Ecological Risk Assessments (ERA) most commonly use data on individual organisms due to the infeasibility of large, ecosystem or population-level experiments. Therefore, the extrapolation of stressor effects from the individuallevel to higher levels of biological organization is of great importance when considering the potential effect of a stressor, specifically a contaminant in ecotoxicological applications. In this manuscript, we build on previous work (Stevenson et al. 2017 $)$ in which we predicted from data on individual life histories a threshold daily food requirement such that populations of Daphnia pulicaria exposed to silver nanoparticles (AgNPs) at one concentration will persist. We conducted an experiment exposing daphnid populations to the same concentration of AgNPs at which the individual-level data would predict persistence at all but the lowest food input rate which was close to the extinction threshold. We fit a bioenergetic model to the individual-level data published in Stevenson et al. 2017 and used these parameters in an Individual Based Model (IBM) which predicted population outcomes broadly matching those observed. This work highlights the importance of studies on individuals at ecologically plausible food levels. It also points to the importance of considering ecological feedbacks, specifically via resource dynamics, when making predictions across levels of biological organization.

We suggest the following reviewers for this manuscript: Drs. Christopher Salice (csalice@ towson.edu), Steven Railsback (steven.railsback@humboldt.edu), Nika Galic (nika.galic@syngenta.com), Nina Marn (Nina.Marn@irb.hr), and Andre Gergs (andre.gergs@bayer.com).

Thank you very much for your consideration of our manuscript. We are excited about the special issue of this journal and think our work is a good fit for its aims, specifically the topics "Bioenergetic or other process-based models that improve characterization of contaminant accumulation and effects" and "Models capable of extrapolating effects across biological scales (e.g., molecular to whole organism to population)".

Sincerely, Louise Stevenson, $\mathrm{PhD}$

Associate Researcher; Biodiversity and Ecosystem Health Group Environmental Sciences Division; Oak Ridge National Laboratory stevensonlm@ornl.gov

\footnotetext{
${ }^{1}$ Stevenson, L. M., Krattenmaker, K. E., Johnson, E., Bowers, A. J., Adeleye, A. S., McCauley, E., \& Nisbet, R. M. (2017). Standardized toxicity testing may underestimate ecotoxicity: Environmentally relevant food rations increase the toxicity of silver nanoparticles to Daphnia. Environmental toxicology and chemistry, 36(11), 3008-3018.
} 


\section{Extrapolating contaminant effects from individuals to populations: a case \\ 2 study on nanoparticle toxicity to Daphnia}

4 Authors: Louise M. Stevenson ${ }^{1,2^{*}}$, Katherine E. Krattenmaker ${ }^{2}$, Edward McCauley ${ }^{3}$, Roger M.

5 Nisbet $^{2}$

6 Environmental Sciences Division, Oak Ridge National Laboratory, Oak Ridge, Tennessee, USA

$7{ }^{2}$ Department of Ecology, Evolution and Marine Biology, University of California, Santa Barbara, 8 California, USA

$9 \quad{ }^{3}$ Department of Biological Sciences, University of Calgary, Calgary, Alberta, Canada

10 *corresponding author, stevensonlm@ ornl.gov, ORCID ID: 0000-0003-4967-9897

12 Note: This manuscript has been authored by UT-Battelle, LLC under Contract No. DE-AC05-

13 00OR22725 with the U.S. Department of Energy. The United States Government retains and the

14 publisher, by accepting the article for publication, acknowledges that the United States

15 Government retains a non-exclusive, paid-up, irrevocable, world-wide license to publish or

16 reproduce the published form of this manuscript, or allow others to do so, for United States

17 Government purposes. The Department of Energy will provide public access to these results of

18 federally sponsored research in accordance with the DOE Public Access Plan

19 (http://energy.gov/downloads/doe-public-access-plan).

\section{Acknowledgements:}

22 We thank B. Martin and E. Muller for helpful discussion and C. Briggs, P. Holden, and 23 anonymous reviewers for comments on the manuscript. We also thank E. Wear for help with

24 DAPI staining. The present study was supported by the US National Science Foundation

25 (http://www.nsf.gov/) and the US Environmental Protection Agency (EPA, http://www.epa.gov/)

26 under Cooperative Agreement EF-0830117, EPA's Science to Achieve Results grant R835797,

27 and The Worster Family Research Award for undergraduate-graduate student research and

28 mentorship at the University of California, Santa Barbara. The funders had no role in study

29 design, data collection and analysis, decision to publish, or preparation of the manuscript. 
Abstract:

Ecological risk assessment (ERA) is charged with assessing the likelihood a chemical

33 will have adverse environmental or ecological effects. When assessing the risk of a potential

34 contaminant to biological organisms, ecologists are most concerned with the sustainability of populations of organisms, rather than protecting every individual. However, ERA most commonly relies on data on the effect of a potential contaminant on individuals because these experiments are more feasible than costly population-level exposures. In this work, we address the challenge of extrapolating these individual-level results to predict population-level effects.

39 Previous per-capita population growth rate estimates calculated from individual-level exposures of Daphnia pulicaria to silver nanoparticles (AgNPs) at different food rations predict a critical

41 daily food requirement for daphnid populations exposed to $200 \mu \mathrm{g} / \mathrm{L}$ AgNPs to avoid extinction.

42 To test this, we exposed daphnid populations to the same AgNP concentration at three different

43 food inputs, with the lowest ration close to the extinction threshold predicted from data on

44 individuals. The two populations with the higher food inputs persisted, and the population with

45 the lowest food input went extinct after 50 days but did persist through two generations. We

46 demonstrate that we can extrapolate between these levels of biological organization by

47 parameterizing an individual-level biomass model with data on individuals' response to AgNPs

48 and using these parameters to predict the outcome for control and AgNP-exposed populations.

49 Key to successful extrapolation is careful modeling of temporal changes in resource density,

50 driven by both the experimental protocols and feedback from the consumer. The implication for

51 ecotoxicology is that estimates of extinction thresholds based on studies of individuals may be

52 reliable predictors of population outcomes, but only with careful treatment of resource dynamics.

54 Introduction: 
One important goal of ecological risk assessment (ERA) of chemicals is to estimate the

56 effects of a potential contaminant on populations, however population experiments are costly in

57 terms of labor, materials, and organisms. In practice, ERAs often end up relying on data from

58 experiments on individuals, and, in the case of ERAs for freshwater systems, they commonly use

59 data from chronic toxicity tests (OECD 2012) in which Daphnia are exposed to increasing

60 concentrations of a potential toxicant and their survival and reproduction are measured. From

61 these individual-level data, it is possible to estimate metrics related to population-level

62 responses, such as the long-run population growth rate in a constant environment (per-capita

63 population growth rate, $r$ ), an approach that has been employed to predict the effect of toxicants

64 on populations from individual level data for many years (Kooijman and Metz 1984). However,

65 such metrics do not directly permit prediction of population-level impacts, unless they take

66 account of potentially important feedbacks between the individuals that comprise the population

67 and their environment. Recognition of this limitation has inspired calls for the use of ecological

68 modeling to incorporate ecological complexity into ERA (Forbes et al. 2011; Forbes et al. 2009).

69 The feasibility, and challenges, of the use of models to extrapolate ecotoxicological impacts

70 across levels of biological organization was demonstrated for Daphnia by (Martin et al. 2013a;

$71 \quad$ Martin et al. 2013b).

72 Our past work (Stevenson et al. 2017) studied the effect of silver nanoparticles (AgNPs) on

73 individual Daphnia fed a range of algal food rations (mgC algal food per daphnid per day). We

74 found that low food increased AgNP toxicity, and that two AgNP exposures (75 and $200 \mu \mathrm{g} / \mathrm{L})$

75 had direct effects on daphnid survival (Stevenson et al. 2017). Specifically, $200 \mu \mathrm{g} / \mathrm{L}$ AgNPs was

76 overwhelmingly toxic at all but the highest food ration with no individuals exposed to $200 \mu \mathrm{g} / \mathrm{L}$

77 surviving to reproduce except two at the highest food ration. AgNPs are the most studied 
nanoparticles when it comes to the toxicity of nanoparticles to Daphnia, and, according to a

79 recent review, the most susceptible to AgNP toxicity (Liu et al. 2022). Other studies have

80 similarly found that AgNPs are toxic at low, parts per billion concentrations to Daphnia (Garner

81 et al. 2015; Griffitt et al. 2008; Hoheisel et al. 2012; Qin et al. 2015; Ribeiro et al. 2013;

82 Stensberg et al. 2014; Ulm et al. 2015), including sublethal effects on growth and/or

83 reproduction (Mackevica et al. 2015; Ribeiro et al. 2013; Sakamoto et al. 2015; Zhao and Wang

84 2011). For population predictions, the most important finding from Stevenson et al. (2017, see

85 their Fig. 5)) was identifying a critical combination of food ration per daphnid and stressor

86 concentration for a contaminated population to be viable ( $r$ close to zero).

87 To empirically test our interpretation of our data on individuals, we exposed small

88 populations of Daphnia pulicaria to $200 \mu \mathrm{g} / \mathrm{L}$ AgNPs at three food inputs and developed an

89 Individual Based Model (IBM) that, apart from one calibration, utilized only parameters

90 estimated from our individual-level data or supporting literature. We then simulated the

91 population-level impact of AgNPs on these daphnid populations.

92 IBMs, also known as Agent Based Models, track individuals through time and population

93 dynamics emerge as these individuals interact with each other and their shared environment.

94 Multiple studies have used IBMs to extrapolate individual-level effects to the population-level

95 for ecological (Martin et al. 2013a; Railsback and Grimm 2019) and ecotoxicological

96 applications (Martin et al. 2013b; Preuss et al. 2010). Some studies have integrated IBMs into

97 larger ecosystem models to extrapolate effects to higher levels of biological organization (Bartell

98 et al. 2019; Forbes et al. 2019; Schmolke et al. 2019). Here, we incorporate a generalizable net

99 production model (a variant of the model in Nisbet et al. 2004) into an IBM framework with

100 modules to describe algal food and damage dynamics to connect individual to the population- 
101 level effects of AgNPs we observed empirically. In our population experiments reported here, we

102 found that $200 \mu \mathrm{g} / \mathrm{L}$ AgNPs did not have a strong effect on populations of Daphnia except at the

103 lowest food input after 50 days. The IBM we developed connects the individual and population

104 levels: an IBM parameterized using the individual-level data and supporting literature was able

105 to predict the equilibrium population biomasses observed in our experiments. This work

106 highlights the predictive power of detailed studies of growth, reproduction, and mortality for the 107 extrapolation of toxicant effects from the individual to population level.

109 Methods: Experimental

\section{Silver nanoparticles characterization}

111 We purchased $40 \mathrm{~nm}$ BioPure citrate-coated silver nanoparticles (AgNPs) from

112 NanoComposix (San Diego, CA). We measured the size, dissolution, and reactive oxygen

113 species (ROS) production of AgNPs in the light and in the dark (since these daphnid experiments

114 were performed in the dark to control algal growth which would change the food concentration)

115 and these data are reported in Stevenson et al. 2017. In summary, AgNPs at these exposures

116 concentrations do not produce ROS, do no aggregate, and have very slow dissolution in the "low

117 P" COMBO media (Kilham et al. 1998) used for the experiments reported here. Our data

118 indicate that less than $1 \mu \mathrm{g} / \mathrm{L}$ of the $200 \mu \mathrm{g} / \mathrm{L} \mathrm{AgNP}$ exposure is present after three days (the

119 longest transfer interval) as ionic silver (Stevenson et al. 2017).

\section{Daphnia individual experiments}

122 We exposed individual Daphnia pulicaria to $200 \mu \mathrm{g} / \mathrm{L}$ AgNP and fed them four different

123 food rations, as detailed in Stevenson et al. 2017. As described in that paper, these exposures 
124 were comprised of multiple experiments: an experiment at the highest food ration (0.01

$125 \mathrm{mgC/daphnid/day,} \mathrm{which} \mathrm{ran} \mathrm{for} 26$ days) and two experiments at the other food rations (which

126 ran for the entire lifespan of the organisms). We conducted two experiments at the food rations

$1270.0005,0.001$, and $0.0025 \mathrm{mgC} /$ daphnid/day: the first experiment consisted of control and AgNP

128 exposures ( $\mathrm{n}=8$ for each treatment) and the second experiment consisted of six additional

129 control individuals. We started additional control individuals because survival at the lower food

130 rations was initially lower than we expected, and we wanted to ensure we had enough data to

131 parameterize the control response of $D$. pulicaria to these lower food conditions.

\section{Daphnia population experiment}

134 To test the effect of AgNPs at different food inputs to populations of Daphnia, we set up 135 twelve small populations, half of which were kept as controls and half of which were dosed with $136200 \mu \mathrm{g} / \mathrm{L}$ AgNPs. The populations were fed one of three food inputs $(0.07,0.14$, and 0.27

$137 \mathrm{mgC/day})$, and there were two replicate populations per treatment. Populations were kept in 400 138 mL of autoclaved "low P" COMBO media (Kilham et al. 1998) and all glassware was autoclaved 139 prior to the start of the experiment.

140 We started the populations at the approximate neonate:adult ratio of Daphnia pulicaria

141 populations at equilibrium, based on data from past in experiments in our lab (unpublished data).

142 This ratio is approximately 3 neonates or juveniles: 1 adult and we started the populations with 143 small neonates $(0.69-0.71 \mathrm{~mm})$, large neonates $(0.71-0.81 \mathrm{~mm})$, and adults $(2.0 \pm 0.5 \mathrm{~mm})$.

144 We removed Daphnia from our stock tanks 5 days prior to the experiment and placed them in 145 tanks of fresh COMBO media for two days, then transferred them to another tank of fresh 146 COMBO media for another 3 days to clean the individuals and minimize any carryover of algal 
147 cells or other detritus into the experimental containers. We started the populations at total

148 populations sizes proportional to the food input; the populations fed the highest food input $(0.27$

$149 \mathrm{mgC/day}$ ) started with the most individuals (25 day-old neonates, 9 large neonates, and 11

150 adults), the middle food input started with approximately half that number of individuals (5 day-

151 old neonates, 13 large neonates, and 5 adults), and the lowest food input started with

152 approximately a quarter of the population size as the highest food input (2 day-old neonates, 7

153 large neonates, and 3 adults).

154 We sampled each population on a Monday-Wednesday-Friday schedule, resetting algal

155 food densities, media, and AgNP exposure every 2, 2, and 3 days. For sampling, we poured the

156 daphnid populations onto a 60-micron nylon net filter (Millipore NY60) and counted and

157 identified the stage of all individuals using a dissecting scope (Leica M80). We identified

158 developmental stages of the individuals under the microscopes using circles printed on a

159 transparency film of known diameters to correspond to the stages of interest. There were two

160 circles: neonates fit into the circle with diameter $1.0 \mathrm{~mm}$, juveniles fit into the circle with

161 diameter $1.8 \mathrm{~mm}$, and any individuals larger than both circles were adults. We also identified

162 pregnant adults and counted the number of eggs and embryos in the brood pouch. We then

163 placed the individuals into fresh COMBO media dosed with 0 or $200 \mu \mathrm{g} / \mathrm{L} \mathrm{AgNPs}$ and fed the

164 populations.

165 We fed the Daphnia Chlamydomonas reinhardtii cells from cultures 10-12 days old. We 166 centrifuged a $500 \mathrm{~mL}$ algal batch culture on 7,000 rpm for 4 minutes, re-suspended the cells in

167 nanopure water, and then measured the concentration of chlorophyll fluorometrically (see

168 methods in Stevenson et al. 2013). We then converted the chlorophyll a concentration to a

169 carbon concentration using a fixed $\mathrm{mg} \mathrm{C}: \mu \mathrm{g}$ chlorophyll ratio that was measured empirically in 
170 our lab $(0.22 \mathrm{mgC} / \mu \mathrm{g}$ chlorophyll a). We measured the concentration of algal carbon fed through

171 time by removing $5 \mathrm{~mL}$ of sample and drying the samples down in a drying oven and analyzing

172 them for total carbon and nitrogen on a CN analyzer (Thermo Scientific Flash $200 \mathrm{CN}$

173 Analyzer). We also measured the amount of food left behind by the populations on a microplate

174 reader and converted these concentrations to chlorophyll a concentrations (see methods in

175 Stevenson et al. 2013).

176 We placed the experimental populations in the dark for the duration of the experiment to

177 maintain the fed algal food input. The cultures were at a temperature of $21.8 \pm 0.6$ degrees

178 Celsius (average \pm standard deviation of hourly measurements taken by Maxim Integrated

179 iButton DS1921G throughout experiment placed in $400 \mathrm{~mL}$ of water next to the experimental

180 cultures). The experiment was run for 89 days for all treatments except the highest food input,

181 AgNP treatments - those were concluded on Day 68.

182

\section{Bacterial cell counts}

184 Throughout the experiment, we noticed that AgNP-exposed populations had more

185 buildup of detritus, especially shed carapaces, compared to controls, potentially indicating

186 AgNPs may be toxic to the bacterial populations in our experiment and hindering breakdown of

187 organic material. We measured the bacterial population through DAPI staining (4',6-diamidino-

188 2-phenylindole), a commonly used method for counting cells as the stain binds to DNA and

189 fluoresces. We removed $50 \mathrm{~mL}$ samples from media post-transfer (we removed the daphnid

190 individuals and then took samples of the media left behind), fixed them with formalin, and then

191 stained the samples within 72 hours of collection. We stained $5 \mathrm{~mL}$ of each sample with $0.5 \mathrm{~mL}$

192 of $5 \mu \mathrm{g} / \mathrm{mL}$ DAPI (Acros Organics) for 3 minutes and then filtered via a vacuum filtration 
193 system. We filtered the samples through 0.2 micron PC filters (Whatman Nuclepore

194 Polycarbonate Track-Etched Membrane) stained with irgalan black ( $2 \mathrm{mg} / \mathrm{mL})$ with a 0.8 micron

195 backing filter (Fisherbrand General Filtration Membrane Filter) behind the 0.2 micron filter. We

196 mounted the filters onto glass microscope slides and counted bacterial cells using

197 epifluorescence microscopy (Olympus B202).

199 Methods: theory

200 Individual model description

201 We fit a variant of the net production biomass-based model of individual daphnid growth

202 and reproduction from Nisbet et al. 2004 to the data in Stevenson et al. (2017) on the effect of 203 AgNPs on D. pulicaria fed different food rations (Table 1). The only difference from Nisbet et 204 al. (2004) was that we assumed ingestion rate of food for individuals is proportional to carbon 205 mass rather than use a spline-based function as in the published paper. As our target population 206 data came from transfer cultures where all food is eaten or sinks to the bottom of the culture flask

207 (see below), this modification is expected to have small impact on model predictions of biomass 208 and greatly eases fitting. It is, however, important to note that the omission of any allometry in 209 net production removes any characterization of juvenile-adult competition for food which is

210 likely critical for determining changes in population demography, notably any tendency to

211 cycling (de Roos and Persson 2013). It thus also likely eliminates the likelihood that the IBM

212 will correctly characterize the dynamics early in the experiment - even in biomass - where we

213 anticipate the competition among individuals in the initial inoculum will be important. For this

214 reason, our model tests focused on the biomass dynamics near the end of the population

215 experiments. 
216 The model has three state variables - algal food density, carbon weight of an individual, and

217 the cumulative number of eggs produced. The model distinguishes between juveniles and adults

218 - any individual less than the weight at reproductive maturity (estimated from the individual

219 data) is a juvenile, and any individual above that weight is an adult. The transition to the "adult"

220 stage occurs at the size where the $D$. pulicaria started releasing free-swimming neonates; we

221 made this decision because our individual and population-level models do not include explicit

222 molts or any delay between energy committed to reproduction and the production of neonates.

223 Since our experiments were transfer cultures (where the media and food were replaced on a

224 Monday-Wednesday-Friday schedule), the algal food densities resets to the food ration every 2 ,

2252 , and 3 days. In between the transfer intervals, daphnid individuals feed at a rate proportional to

226 their weight and with a Type 2 functional response to instantaneous food density. Maintenance

227 rate per unit of body weight is the same for all stages. Adults allocate assimilated carbon to either

228 growth and maintenance or to reproduction, and the proportion of carbon allocated to

229 reproduction is defined by the function . Juveniles do not allocate any carbon to reproduction.

230 Adults produce eggs at a rate proportional to the amount of carbon assimilated that is allocated to

231 reproduction, and this amount of carbon is converted to eggs based on the amount of carbon that

232 is required to produce one egg ( ). Individuals in this model were allowed to shrink in terms of

233 weight but could not re-utilize energy already previously allocated towards reproduction.

234 We initialized the model with all individuals starting at the weight at birth $\left(W_{b}\right)$. We

235 converted the measured length data to carbon weight using the equation in Paloheimo et al.

236 (1982) for D. pulex $\left(\mathrm{W}=0.00624 \mathrm{~L}^{2.4}\right.$ for $\mathrm{W}=$ weight in $\mathrm{mg}$ dry weight and $\mathrm{L}=$ body length in

$237 \mathrm{~mm}$ ). We previously verified that this relationship is consistent with data on D. pulicaria 
238 (unpublished data), a species with very similar physiological parameters. We converted dry

239 weight to mgC assuming that the daphnid is $42 \%$ carbon (unpublished data on D. pulicaria).

\section{Population model description}

We modeled the dynamics of the empirical populations using an Individual Based Model

243 (IBM) coded in NetLogo (version 6.2). Growth and reproduction of individual Daphnia were

244 modeled using the net production model (Table 1) with parameters fit to the individual-level data

245 from Stevenson et al. 2017 (Table 2) with a few additional parameters defined by the

246 experimental setup or fit to the individual-level data (Table 3). The simulated populations are

247 transferred into fresh food on Mondays, Wednesdays, and Fridays (any food leftover from the

248 previous feeding is removed and food is supplied at the food supply rate for that population).

249 Individuals release a neonate after allocating the appropriate amount of carbon for reproduction.

250 New offspring are initialized with $0.0011 \mathrm{mgC}$. Any energy dedicated to egg production leftover

251 after offspring release is saved until the next molt. Two new modules were added: a one-

252 parameter "damage" module describing age and toxicant impacts on individual hazard rate (risk

253 of death per unit time) and a module describing algal "sinking" (also called settling) to the

254 bottom of the culture flasks where they become much less accessible to daphnids. We assumed

255 that the sole physiological mode of action of the AgNPs is on mortality.

The IBM is initialized using the number and carbon mass of individuals that started the

257 experiments which varied between the food supply rates (see earlier methods section). All

258 individuals started without eggs. The populations were simulated for up to 89 days or until all

259 individuals died and each food input and treatment (AgNP and control) was simulated 100 times

260 because of the stochastic mortality term (discussed below). 
The damage module is a simplified implementation of an approach to mortality estimation

262 now widely used in ecotoxicology, notably in applications using GUTS (e.g. Jager et al. (2011)).

263 In brief, we couple a maximally simple characterization of toxicokinetics, and assume that in

264 response, an organism accumulates physiological "damage", an abstract concept that potentially

265 encompasses a vast range of biological mechanisms. Hazard rate is assumed to be proportional to

266 damage. Here, we assume that Daphnia individuals absorb AgNPs at a rate proportional to their

267 biomass and define $Q=$ body burden of AgNP, $W=$ biomass, $q=Q / W=$ damage density.

268 Uptake rate of AgNP is assumed proportional to biomass and we assume no loss or

269 transformation of absorbed AgNP.

270

$$
\text { Then } \frac{d Q}{d t}=a W \text { and } \frac{d q}{d t}=a-\underbrace{q\left(\frac{1}{W} \frac{d W}{d t}\right)}_{\text {uptake }}=a-g(t) q \text { with } g=\frac{1}{W} \frac{d W}{d t}
$$

The immediate implication is that damage density increases faster when growth is slower.

273 transfer of AgNP, so that for all individuals $q$ is zero at birth. A mathematical consequence of

274 this assumption is that the ODE for $q$ has a solution of the form

$$
q(t)=a \times \text { function independent of } a \text { involving growth history }
$$

276 We have no data on bioaccumulation and therefore cannot estimate the parameter $a$. However, we can rescale and work with a differential equation for the ratio $q / a$, (which has units of time)

278 and which can be interpreted as a damage index. If $\tau=\frac{q}{a}$, then

$$
\frac{d \tau}{d t}=1-g(t) \tau
$$

280 Hazard rate $\mu$ is now assumed to be the sum of a background and a term proportional to damage 281 density and we can thus set 


$$
\mu=\mu_{0}+A \tau
$$

283 where A is a single parameter estimable from the data in Stevenson et al. (2017).

We incorporated an algal sinking module into the IBM because we found that the

285 populations only consumed roughly half of the algal food they were provided. In the algal

286 sinking module, the $C$. reinhardtii cells sink with an exponential rate $\alpha$ such that the cells are no

287 longer available to the Daphnia when they have sunk to the bottom of the container.

\section{Methods: statistical}

290 We estimated parameters in a series of steps, executed sequentially.

291 1) Estimate control parameters for the model of growth and reproduction.

292 2) Assume that the population experimental results are impacted by sinking algae using a 293 combination of data from our experiments and from published literature.

294 3) Estimate background mortality for controls using data from Stevenson et al. (2017) and modify with an estimate of the damage related parameter $A$ by calibrating the estimated long run growth rate and comparison with Fig. 5 of Stevenson et al. (2017)

4) For $200 \mu \mathrm{g} / \mathrm{L}$ treatments, estimate the new parameter $A$ by calibrating to fit the $200 \mu \mathrm{g} / \mathrm{L}$

299 We describe each below.

300 1) We fixed parameters $\varepsilon$ and to values based on well-established estimates in the 301 literature (summarized in Nisbet et al. (2004)). We calculated the value of $b$ using the 302 data in Stevenson et al. 2017, specifically growth at the food input of 0.001 $303 \mathrm{mgC/daphnid/day.} \mathrm{At} \mathrm{this} \mathrm{food} \mathrm{ration,} \mathrm{Daphnia} \mathrm{did} \mathrm{not} \mathrm{reproduce} \mathrm{and} \mathrm{growth} \mathrm{saturated}$ 304 around $1.9 \mathrm{~mm}(0.0122 \mathrm{mgC})$. We assumed an assimilation efficiency of 0.7 , such that 
the Daphnia were assimilation approximately $0.0007 \mathrm{mgC} / \mathrm{daphnid} / \mathrm{day}$. Assuming all of that energy went towards growth and respiration (since the individuals did not reproduce), we calculated a value of the maintenance rate (b) to be $0.061 /$ day, a value consistent with past studies of Daphnia. We estimated the remaining parameters using a likelihood method coded in the package BYOM ("Bring Your Own Model”) platform for parameter estimation, developed by Tjalling Jager (http://debtox.info) for Matlab (Mathworks). Details on the fitting routines in this software can be found in Jager (2021). We estimated the values of parameters $I, F_{h}$, and $\rho$ by fitting all food levels simultaneously. Negative log likelihood values the model fit is provided in the figure legend. Confidence intervals were calculated using likelihood profiles (95\% probability from the $\chi^{2}$ distribution).

2) Data from measurements of residual algae yielded an estimate of 2.0 day $^{-1}$ for the sinking rate $\alpha$. Given our large uncertainty this estimate is consistent with a published estimate of $1.57 \mathrm{day}^{-1}$ (Kearns and Hunter 2001). We used this published value in the simulations reported here.

3) We estimated the background mortality rate using the individual-level data (Stevenson et al. 2007), assuming a constant, exponential decay function. We estimated the value of the damage accumulation rate for controls by adjusting the value of $A$ until the final biomass in population controls with the highest food input was close to the equilibrium biomass we found in the population experiments. We then tested this value of $A$ by computing the long-term population growth rate $(r)$ applying the Euler-Lotka equation to simulations of the individual experiments with food inputs 0.01 and $0.0025 \mathrm{mgC} / \mathrm{daphnid} /$ day and comparing with the values calculated by (Stevenson et al. 2017 Fig. 5). 
4) We used this method to estimate the value of $A$ for the AgNP treatment - we adjusted the value of $A$ until the calculated value for $r$ matched the values found in Stevenson et al. 2017. For the AgNP treatments, only individuals at the highest food ration produced neonates, so only one value of $r$ could be used. The damage accumulation rate for the AgNP treatment was estimated by matching this estimate of $r$.

334 calculate mortality rates for the IBM, we performed linear regression using R (version 3.2.3). To

335 calculate average background mortality rates, we assumed survival followed an exponential 336 decay function and calculated the slope of this function by taking the natural log of survival and

337 time and calculating the slope of the resulting line. Confidence intervals were calculated in $\mathrm{R}$ 338 using 'confint'.

Results: Parameter estimates

\section{Individual-level model parameter values}

We fed the Daphnia algal food at the nominal food rations $(0.0005,0.001,0.0025,0.01$ mgC/daphnid/day) assuming a fixed chlorophyll to carbon ratio (Stevenson et al. 2017). We

344 measured the carbon concentration of food fed throughout the experiment and the actual 345 concentrations fed were $0.00064,0.0013,0.0032$, and $0.0128 \mathrm{mgC} /$ daphnid/day (unpublished 346 data). We used the measured values converted to an algal carbon density at the start of each 347 transfer interval as the model food inputs (Figure 2). The fit of the individual-level model to our individual level data is shown in Figure 2.

349 The model broadly matched the dynamics of growth and reproduction in D. pulicaria fed a wide 350 range of food rations. There are some significant mismatches, specifically the model 
351 underpredicts growth at the lower food rations but recognizing the importance of reproduction

352 for population dynamics, we accepted this limitation of our individual model.

\section{Population-level model parameter values}

355 The IBM required a few parameters not estimated for the individual-level model,

356 specifically the background hazard rate, damage accumulation rate, and algal sinking rate. We

357 also explicitly measured concentration of algal carbon fed to the populations and used these

358 values for the food input rate.

\section{Hazard model}

361 We calculated background hazard rate for unexposed individuals across the food rations

362 (Figure S1) assuming that the individuals die exponentially. The parameter value and confidence 363 interval are reported in Table 3.

364 We estimated the values for the damage accumulation rates for control and AgNP365 exposed individuals and these values are reported in Table 3. We estimated these values by 366 comparing the predicted $r$ values for these estimates of $A$ compared to the calculated $r$ values in

367 Stevenson et al. 2017. Values were only calculated for the two highest food rations, 0.01 and $3680.0025 \mathrm{mgC} / \mathrm{daphnid} / \mathrm{day}$, because those are the only two food rations at which the Daphnia 369 reproduced, and no individuals exposed to AgNPs reproduced at food rations less than the 370 highest. In the controls, the $r$ values calculated using the estimate of $A$ reported here are 0.042

371 and 0.20 1/day for 0.0025 and $0.01 \mathrm{mgC} /$ daphnid/day, respectively. For the AgNP-exposed

372 individuals, the $r$ value calculated using the estimate of $A$ reported here is 0.18 per day for 0.01

$373 \mathrm{mgC} / \mathrm{daphnid} / \mathrm{day}$. All of these values are within the variation reported in Stevenson et al. 2017. 
375 Algal sinking rate

376 We found that the daphnid populations did not consume $18-63 \%$ of the algal food they

377 were fed, and this difference varied across the number of transfer days that had elapsed and, to a

378 lesser extent, between the control and AgNP treatments (Figure S2). We hypothesize that this is

379 due to algal sinking, which we explicitly added to the IBM as described in the Methods section.

380 Kearns and Hunter (2001) measured the sinking or settling rate of C. reinhardtii

381 spectrophotometrically and found a value of 1.57 1/day. When plugged into the algal sinking

382 module, this value roughly predicts the concentration of algal food left behind we observed in

383 our experiment (Figure S2). With this value of $\alpha$, the algal sinking model predicts: at the highest

384 food input, the daphnid populations would eat roughly $50 \%$ and $40 \%$ of the algal food after 2 and

3853 transfer days, respectively; at the medium food input, the daphnid populations would eat

386 roughly $40 \%$ and $30 \%$ of the algal food after 2 and 3 transfer days, respectively; and at the

387 lowest food input, the daphnid populations would eat roughly $40 \%$ and $36 \%$ of the algal food

388 after 2 and 3 transfer days, respectively. An obvious deviation between our model and data

389 (Figure S2) is that the model predicts that the daphnid populations would eat less of the food

390 after the 3-day than the 2-day transfer interval, however our data show that the populations ate

391 more of the food after the longer transfer interval. This is probably due to the daphnid

392 populations starting to root around at the bottom of the beaker after an extended period without

393 food, possibly eating some of the algal cells that the model assumes are unavailable because they

394 have sunk. Although there is this mismatch, this simplified model using the single parameter

395 value from Kearns and Hunter (2001) broadly captures the algal food dynamics observed in our

396 population experiments. 
Algal food input concentrations

We assumed the same fixed chlorophyll to carbon ratio as discussed for the individual-

400 level data in order to calculate the concentration of algal food to feed the population exposures.

401 We also measured the algal food throughout the experiment (Figure S3) and found that the

402 concentration varied across the experiment. We used the average of these values as the input for

403 the IBM: the concentration of algal food fed was $0.168,0.34$, and $0.675 \mathrm{mgC} /\left(\mathrm{L}^{*}\right.$ day) (Table 3).

\section{Results: Bacterial measurements}

406 The tested concentration $(200 \mu \mathrm{g} / \mathrm{L})$ of AgNPs has only a small effect on bacterial

407 communities. We measured the bacterial population in all experimental cultures after a 2- and 3-

408 day transfer interval to analyze for differences between AgNP and control cultures. We found no

409 difference between control and AgNP bacterial populations after the three-day transfer interval.

410 (Figure S4A), however samples taken after the two-day transfer interval were significantly

411 different (Figure S4B). The food input $(\mathrm{p}=0.011)$ and $\operatorname{AgNP}$ treatment $(p=0.010)$ both had

412 significant effects on bacterial abundance.

413 These samples were taken on days 57 and 59 of the population experiment, as AgNP-

414 exposed populations were recovering from the initial decline around day 50. It is unclear whether 415 the significant or the not-significant result from the two- and three-day transfer results,

416 respectively, more accurately describes the average state of the cultures throughout the

417 experiment. However, $200 \mu \mathrm{g} / \mathrm{L}$ AgNPs is probably not a high enough concentration to kill most

418 bacterial species - a meta-analysis of AgNP toxicity studies to a variety of taxa found that the

419 average median lethal concentration (LC50) or half maximal effective concentration (EC50) of 
420 AgNPs to bacteria is $7.10 \mathrm{mg} / \mathrm{L}$, about 35 times higher than our exposure concentration

421 (Bondarenko et al. 2013). A more recent review of silver nanoparticle toxicity found that EC50

422 or minimum inhibitory concentration (MIC) values of AgNPs to bacteria ranged from $0.27-250$

$423 \mathrm{mg} / \mathrm{L}$ with an average of $23.24 \mathrm{mg} / \mathrm{L}$ (Liu et al. 2022).

425 Results: Population impacts of exposure to AgNPs

$200 \mu \mathrm{g} / \mathrm{L}$ AgNPs only impact populations substantially after 50 days to lowest food input

The tested concentration $(200 \mu \mathrm{g} / \mathrm{L})$ of AgNPs had no significant effect on the two

428 highest food inputs, and only had an effect on populations fed the lowest food input after 50 days

429 (Figures 1, 3, 4, S5-9). We can conjecture that the delay in responding is because the population

430 starts with the "luxury" of undamaged adults who can produce many neonates before

431 accumulating large levels of damage. The next generation starts accumulating damage from birth

432 and hence has a much lower change of surviving to reproduce. Although there is some

433 variability, there are no large differences between control and AgNP treatments in the two

434 highest food inputs (Figure 1). After 50 days, all AgNP-exposed treatments decline across all

435 food inputs. This effect is stronger at lower food inputs - at $0.27 \mathrm{mgC} / \mathrm{day}$, the population returns

436 to control levels within 10 days and do not decline as sharply, while populations fed 0.14

$437 \mathrm{mgC} /$ day take about 15 days to return to control levels, and populations fed the lowest food input

438 decline to extinction (Figure 1). Populations exposed to AgNPs at the two highest food inputs

439 were able to recover to control levels, driven by boosts in fecundity in populations fed 0.14 and

$440 \quad 0.27 \mathrm{mgC} /$ day between days 50 and 70 (Figure S9), however populations fed the lowest food

441 input never recovered. One AgNP-exposed replicate at the lowest food input went extinct

442 immediately on day 54 while the other declined to one adult that continued to produce offspring 
443 every transfer interval, however none of her offspring reached adulthood and the adult eventually

444 died. While there are declines in the AgNP-treatments across all stages (Figures S6-8), adults

445 seem to be the stage most affected by this decline in even the control treatments (Figure S8).

446 As noted earlier, we regard the most reliable test of the model to be biomass levels late in the

447 experiment. Figure 3 displays the carbon biomass of the empirical daphnid populations through

448 time compared to the IBM's prediction of the carbon biomass of the daphnid population (the

449 mean of the values of the carbon biomass of the simulated populations after Day 68 from 100

450 IBM runs and the error bars represent their standard deviation).

452 A population model parameterized with individual data correctly predicts population

453 persistence and long-term biomass.

454 We were able to predict the biomass towards the end of the population experiment using

455 our IBM parameterized mostly using individual-level data (Figure 3). We assumed AgNP

456 bioaccumulation resulted in the accumulation of damage which increased the hazard rate. We get

457 general agreement between the predicted long-term biomasses of the model compared to the data

458 collected from our experiment (Figure 3). Overall, the IBM underpredicted the biomass at the

459 lowest food input in the control treatment but agreed with the middle and highest food inputs for

460 the controls. Interestingly, the opposite was true for the AgNP treatments - the IBM

461 underpredicted the highest food ration but matched the empirical populations better at the two

462 lower food inputs. Importantly, the IBM successfully predicted that daphnid populations exposed

463 to this concentration of AgNPs could go extinct at the lowest food input rate but could persist

464 with more food: out of 100 IBM simulations, 0 , 1, and 81 populations went extinct at the high,

465 middle, and low food inputs, respectively. 


\section{The IBM does not accurately predict the size structure of the empirical populations}

468 As anticipated in the model description, a simplifying assumption in the individual model

469 removes the model's capacity to correctly describe detailed demography. The IBM overpredicts

470 the number of neonates and juveniles present (Figures S11 and S12) and underpredicts the

471 number of adults (Figure S13) at the end of the experiments.

472

\section{Discussion:}

474 Perhaps our most important conclusion for ecotoxicology is that estimation of extinction

475 thresholds utilizing calculations of long run growth rates based on studies on individuals turn out

476 to be reliable predictors of population outcomes. Our population outcome was broadly in line

477 with what would be anticipated from the data on individuals. Stevenson et al. (2017) suggested a

478 threshold with $200 \mu \mathrm{g} / \mathrm{L}$ exposure at around $0.01 \mathrm{mgC}$ per day per animal. Our lowest food

479 population experiments were given a little over 10 times that, with around half of the food

480 sinking. This points to a population in single digits, obviously vulnerable to extinction due to

481 stochasticity. Reliable estimates of $r$ do require individual data over longer time periods that is

482 required in current standardized toxicity tests (OECD 2012), but this is still much less time

483 consuming and expensive than population experiments.

484 A second take home message is that other population metrics are harder to predict. Our

485 predictions of final biomasses in the two viable exposed populations did not match the data well.

486 This is a consequence of our somewhat heroic assumption that we could describe the combined

487 effects of toxicokinetics and toxicodynamics with a single parameter. We also assumed a single

488 physiological mode of action - mortality. 
Our decision to focus on mortality was reached after considerable preliminary work on

490 the individual growth/reproduction data where we assumed AgNPs impacted sublethal processes

491 such that it took more energy for an individual daphnid exposed to AgNPs to grow and

492 reproduce than an unexposed individual. However, after attempting to estimate individual-level

493 parameters to describe this effect, we realized that $200 \mu \mathrm{g} / \mathrm{L} \mathrm{AgNP}$ did not appear to strongly

494 impact sublethal processes, but rather impacted mortality such that AgNP-exposed individuals

495 grew to smaller sizes and did not reproduce as much or at all because individuals died before

496 reaching maturity (Stevenson et al. 2017). This differs from past studies that found that silver

497 decreases reproduction in Daphnia (Sakamoto et al. 2015; Volker et al. 2013), even at

498 concentrations at which mortality was not impacted (Bianchini and Wood 2003; Hook and Fisher

499 2001; Qin et al. 2015; Ribeiro et al. 2013).

Another finding that may have considerable generality is that low food enhances AgNP

501 toxicity to populations of Daphnia. $200 \mu \mathrm{g} / \mathrm{L}$ AgNPs did not have a significant effect on the

502 daphnid populations until around Day 50, when all AgNP-exposed populations declined sharply

503 across all food inputs (Figure 1). Populations at higher food inputs were able to survive the

504 decline in AgNP-exposed populations around day 50 but the populations at the lowest food input

505 went extinct, indicating the compounding effects of nano- and food stress. This conclusion

506 follows our previous work (Stevenson et al. 2017) in addition to other studies that have found

507 that decreased food availability increased the toxic effect of AgNPs (Mackevica et al. 2015;

508 Sakka et al. 2016). This reinforces the message by Stevenson et al. (2017) that in spite of the

509 practical challenges, data on toxicity at ecologically relevant food concentrations (commonly

510 low) is critical. This requires deviation from standardized test standards (OECD 2012) by

511 feeding Daphnia low, environmentally-relevant concentrations of food. 
We end by commenting on the importance of the dynamics of the resource environment

513 for determining population outcomes. One of the more interesting and striking results of this

514 work is that $200 \mu \mathrm{g} / \mathrm{L}$ AgNPs is largely toxic to individuals exposed as neonates at all food

515 rations (Stevenson et al. 2017) but not when the neonates are part of a larger population, except

516 when the food input is very low (Figure 1). We conjecture that this is because the transfer culture

517 regime used in the population experiments resulted in some neonates being released into

518 relatively high food environments. More broadly, the need to consider the dynamics of the

519 resource environment, including feedbacks from an impacted population, needs further emphasis

520 in all considerations of environmental stress. For example, Civitello et al. (in press) recently

521 showed the importance of a snail's resource in determinizing human risk of schistosomiasis from

522 parasite release; under some circumstances removing snails may increase risk. Schmolke et al.

523 (2019) simulated the effect of reduced food availability on a fish population using a coupled

524 IBM and aquatic food web model. Reducing different food items had differential effects on fish

525 population and depended on the fish's diet preferences (Schmolke et al. 2019). Models such as

526 these and the work presented here emphasize the importance of the incorporation of ecological

527 mechanisms when quantifying the impact of anthropogenic change on natural populations. 
Table 1: Individual net production model (variant on Nisbet et al. (2004))

\begin{tabular}{ll}
\hline State variables: & \\
\hline$F(t)$ & Algal food density at time $t(\mathrm{mgC} / \mathrm{L})$ \\
$W(t)$ & Carbon weight of an individual at time $t(\mathrm{mgC})$ \\
$C(t)$ & Cumulative eggs produced at time $t$ \\
\hline Functions: & Size-dependent allocation to growth \\
\hline$\chi=\frac{1}{1+\rho\left(W-W_{p}\right)}$ & Algal food ingestion rate \\
$\phi(t)=\frac{I F W}{F+F_{h}}$ & Weight of an egg \\
$w_{e}=(1+\gamma) W_{b}$ & \\
\hline Balance equations: & Algal food \\
\hline $\begin{array}{l}d F \\
d t\end{array}=-\phi(t) V^{-1}$ & Juvenile growth \\
$\frac{d W}{d t}=\varepsilon \phi(t)-b W$ & Juvenile egg production \\
$\frac{d C}{d t}=0$ & \\
Adults $\left(I f W \geq W_{p}\right)$ & Food \\
$\frac{d F}{d t}=-\phi(t) V^{-1}$ & Adult growth \\
$\frac{d W}{d t}=\chi(\varepsilon \phi(t)-b W)$ & Adult egg production ${ }^{1}$ \\
$\frac{d C}{d t}=\frac{(1-\chi)}{w_{e}}(\varepsilon \phi(t)-b W)$ & \\
\hline
\end{tabular}

532 If maintenance costs were greater than assimilated energy, $\frac{d C}{d t}=0$. 
534 Table 2: Individual model parameters estimated from data in Stevenson et al. (2017) fit to 535 control individuals fed four food rations. We distinguish between $\mathrm{mgC}$ of algae (mgC-A) and $536 \mathrm{mgC}$ of daphnid (mgC-D) biomass.

\begin{tabular}{|c|c|c|c|c|}
\hline & Parameters & Units & Estimate & $\begin{array}{l}\text { Confidence } \\
\text { interval }\end{array}$ \\
\hline I & Maximum specific ingestion rate & $\begin{array}{l}\mathrm{mgC}-\mathrm{A} /(\mathrm{mgC}- \\
\mathrm{D} * \text { day })\end{array}$ & 0.57 & {$\left[\begin{array}{lll}0.55 & 0.60\end{array}\right]$} \\
\hline $\mathrm{F}_{\mathrm{h}}$ & $\begin{array}{l}\text { Half saturation constant in functional } \\
\text { response }\end{array}$ & $\mathrm{mgC}-\mathrm{A} / \mathrm{L}$ & 0.10 & {$\left[\begin{array}{lll}0.094 & 0.10\end{array}\right]^{\mathrm{a}}$} \\
\hline$\rho$ & $\begin{array}{l}\text { Parameter in size-dependent } \\
\text { allocation to growth function }\end{array}$ & $1 / \mathrm{mgC}-\mathrm{D}$ & 1742 & [1364 2246] \\
\hline$\varepsilon$ & Assimilation efficiency & $\mathrm{mgC}-\mathrm{D} / \mathrm{mgC}-\mathrm{A}$ & 0.7 & \\
\hline & Cost of egg production & -- & 0.5 & \\
\hline $\mathrm{b}$ & Maintenance rate & 1/day & 0.06 & \\
\hline$W_{b}$ & Weight at birth & mgC-D & 0.0011 & \\
\hline$W_{p}$ & Weight at puberty & mgC-D & 0.014 & \\
\hline
\end{tabular}

537 aighest value of confidence interval restricted by range across which parameter was allowed to 538 vary. 
Table 3: Parameters for IBM including sources. See text for a description of estimation 541 and methodological details.

542

\begin{tabular}{|c|c|c|c|c|}
\hline & Parameter & Units & Value & Source \\
\hline$\alpha$ & Algal sinking rate & 1/day & 1.57 & $\begin{array}{l}\text { Kearns and Hunter } \\
2001\end{array}$ \\
\hline$\mu_{0}$ & $\begin{array}{l}\text { Background hazard } \\
\text { rate }\end{array}$ & 1/day & $\begin{array}{l}0.04 \\
\text { CI: [ }\left[\begin{array}{ll}0.038 & 0.043\end{array}\right]\end{array}$ & Individual data \\
\hline$A$ & $\begin{array}{l}\text { Damage } \\
\text { accumulation rate }\end{array}$ & 1/day & $\begin{array}{l}0.0005 \text { (Control) } \\
0.005 \text { (AgNP) }\end{array}$ & Individual data \\
\hline V & System volume & $\mathrm{L}$ & 0.4 & $\begin{array}{l}\text { Population } \\
\text { experiment }\end{array}$ \\
\hline$X_{R}$ & $\begin{array}{l}\text { Food concentration } \\
\text { at transfer }\end{array}$ & $\mathrm{mgC} /\left(\right.$ day $\left.^{*} \mathrm{~L}\right)$ & $0.168,0.34,0.675$ & $\begin{array}{l}\text { Population } \\
\text { experiment }\end{array}$ \\
\hline
\end{tabular}


Figures:

549 Figure 1: Total number of individuals in each of the daphnid populations fed different food inputs. Points are data from the daphnid population experiment: data points represent the average and the error bars their standard error. Each treatment (nanoparticle and food concentration) had 2 replicates. Note that the AgNP treatments at the highest food inputs were stopped on Day 68.

Figure 2: Fit of individual model to control Daphnia pulicaria individuals fed four food rations (Stevenson et al. 2017). Food rations are 0.0005, 0.001, 0.0025 and 0.01 $\mathrm{mgC} /$ daphnid/day. Negative log-likelihood value of this fit $=-2323.96$. Data points are averages and error bars represent their standard error.

Figure 3: Equilibrium biomass predictions (horizontal lines) compared to population data. Points are data from the daphnid population experiment: data points represent the average and the error bars their standard error. Each empirical treatment (nanoparticle and food concentration) had 2 replicates. The equilibrium biomass predictions represent the mean of 100 runs of the IBM per treatment (food input and AgNP concentration) and the shaded areas represent the standard deviation of these runs. We defined equilibrium as starting on Day 68 of the empirical and theoretical populations.

Figure 4: Data (points) of total number of individuals in the empirical populations compared to the IBM simulations (lines). Points are data from the daphnid population experiment: data points represent the average and the error bars their standard error. Each empirical treatment (nanoparticle and food concentration) had 2 replicates. The IBM was run 100 times per treatment and then averaged. Error bars represent standard error for the empirical data and standard deviation for the IBM simulations. 


\section{References:}

Bartell SM, Schmolke A, Green N, Roy C, Galic N, Perkins D, Brain R (2019) A Hybrid Individual-Based and Food Web-Ecosystem Modeling Approach for Assessing Ecological Risks to the Topeka Shiner (Notropis topeka): A Case Study with Atrazine. Environmental Toxicology and Chemistry 38(10):2243-2258 https://doi.org/https://doi.org/10.1002/etc.4522

Bianchini A, Wood CM (2003) Mechanism of acute silver toxicity in Daphnia magna. Environmental toxicology and chemistry / SETAC 22(6):1361-1367

Bondarenko O, Juganson K, Ivask A, Kasemets K, Mortimer M, Kahru A (2013) Toxicity of Ag, $\mathrm{CuO}$ and $\mathrm{ZnO}$ nanoparticles to selected environmentally relevant test organisms and mammalian cells in vitro: a critical review. Arch Toxicol 87(7):1181-1200

Civitello D, Angelo T, Nguyen K, Hartman R, Starkloff N, Mahalila M, Charles J, Manrique A, Delius B, Bradley L, Nisbet R, Kinung'hi S, Rohr J (in press) Transmission potential of human schistosomes can be driven by resource competition among snail intermediate hosts. Proc Natl Acad Sci U S A de Roos AM, Persson L (2013) Population and community ecology of ontogenetic development. Princeton University Press, Forbes VE, Calow P, Grimm V, Hayashi TI, Jager T, Katholm A, Palmqvist A, Pastorok R, Salvito D, Sibly R, Spromberg J, Stark J, Stillman RA (2011) Adding Value to Ecological Risk Assessment with Population Modeling. Human and Ecological Risk Assessment: An International Journal 17(2):287-299

Forbes VE, Hommen U, Thorbek P, Heimbach F, Van den Brink PJ, Wogram J, Thulke HH, Grimm V (2009) Ecological models in support of regulatory risk assessments of pesticides: developing a strategy for the future. Integr Environ Assess Manag 5(1):167-172 Forbes VE, Railsback S, Accolla C, Birnir B, Bruins RJF, Ducrot V, Galic N, Garber K, Harvey BC, Jager HI, Kanarek A, Pastorok R, Rebarber R, Thorbek P, Salice CJ (2019) Predicting impacts of chemicals from organisms to ecosystem service delivery: A case study of endocrine disruptor effects on trout. Sci Total Environ 649:949-959

https://doi.org/https://doi.org/10.1016/j.scitotenv.2018.08.344

Garner KL, Suh S, Lenihan HS, Keller AA (2015) Species sensitivity distributions for engineered nanomaterials. Environ Sci Technol 49(9):5753-5759

https://doi.org/10.1021/acs.est.5b00081

Griffitt RJ, Luo J, Gao J, Bonzongo J-C, Barber DS (2008) Effects of particle composition and species on toxicity of metallic nanomaterials in aquatic organisms. Environmental toxicology and chemistry / SETAC 27(9):1972-1978 https://doi.org/10.1897/08-002.1

Hoheisel SM, Diamond S, Mount D (2012) Comparison of nanosilver and ionic silver toxicity in Daphnia magna and Pimephales promelas. Environmental Toxicology and Chemistry 31(11):2557-2563 https://doi.org/10.1002/etc. 1978

Hook SE, Fisher NS (2001) Sublethal effects of silver in zooplankton: importance of exposure pathways and implications for toxicity testing. Environmental toxicology and chemistry / SETAC 20(3):568-574 Jager T (2021) Robust Likelihood-Based Approach for Automated Optimization and Uncertainty Analysis of Toxicokinetic-Toxicodynamic Models. Integr Environ Assess Manag 17(2):388-397 Jager T, Albert C, Preuss TG, Ashauer R (2011) General Unified Threshold Model of Survival a Toxicokinetic-Toxicodynamic Framework for Ecotoxicology. Environ Sci Technol 45(7):2529-2540 
Kearns K, Hunter M (2001) Toxin-producing Anabaena flos-aquae induces settling of Chlamydomonas reinhardtii, a competing motile alga. Microb Ecol 42(1):80-86 Kilham SS, Kreeger DA, Lynn SG, Goulden CE (1998) COMBO: a defined freshwater culture medium for algae and zooplankton. Hydrobiologia

Kooijman SA, Metz JA (1984) On the dynamics of chemically stressed populations: the deduction of population consequences from effects on individuals. Ecotoxicol Environ Saf $8(3): 254-274$

Liu Z, Malinowski CR, Sepúlveda MS (2022) Emerging trends in nanoparticle toxicity and the significance of using Daphnia as a model organism. Chemosphere 291:132941 https://doi.org/https://doi.org/10.1016/j.chemosphere.2021.132941

Mackevica A, Skjolding LM, Gergs A, Palmqvist A, Baun A (2015) Chronic toxicity of silver nanoparticles to Daphnia magna under different feeding conditions. Aquat Toxicol 161:10-16 https://doi.org/10.1016/j.aquatox.2015.01.023

Martin BT, Jager T, Nisbet RM, Preuss TG, Grimm V (2013a) Predicting population dynamics from the properties of individuals: a cross-level test of dynamic energy budget theory. Am Nat 181(4):506-519 https://doi.org/10.1086/669904 Martin BT, Jager T, Nisbet RM, Preuss TG, Hammers-Wirtz M, Grimm V (2013b) Extrapolating ecotoxicological effects from individuals to populations: a generic approach based on Dynamic Energy Budget theory and individual-based modeling. Ecotoxicology https://doi.org/10.1007/s10646-013-1049-x

Nisbet RM, McCauley E, Gurney WSC, Murdoch WW, Wood SN (2004) Formulating and testing a partially specified dynamic energy budget model. Ecology 85(11):3132-3139 OECD (2012) Daphnia magna Reproduction Test. In: Development OfEC-oa (ed). Paris Paloheimo JE, Crabtree SJ, Taylor WD (1982) GROWTH-MODEL OF DAPHNIA. Canadian Journal Of Fisheries And Aquatic Sciences 39(4):598-606

Preuss TG, Hammers-Wirtz M, Ratte HT (2010) The potential of individual based population models to extrapolate effects measured at standardized test conditions to relevant environmental conditions-an example for 3,4-dichloroaniline on Daphnia magna. J Environ Monit 12(11):20702079 https://doi.org/Doi 10.1039/C0em00096e Qin G, Xiong Y, Tang S, Zhao P, Doering JA, Beitel SC, Hecker M, Wang M, Liu H, Lu H (2015) Impact of Predator Cues on Responses to Silver Nanoparticles in Daphnia carinata. Arch Environ Contam Toxicol 69(4):494-505 Railsback SF, Grimm V (2019) Agent-based and individual-based modeling: a practical introduction. Princeton university press, Ribeiro F, Gallego-Urrea JA, Jurkschat K, Crossley A, Hassellov M, Taylor C, Soares AM, Loureiro S (2013) Silver nanoparticles and silver nitrate induce high toxicity to Pseudokirchneriella subcapitata, Daphnia magna and Danio rerio. Sci Total Environ 466467C:232-241 https://doi.org/10.1016/j.scitotenv.2013.06.101

Sakamoto M, Ha J-Y, Yoneshima S, Kataoka C, Tatsuta H, Kashiwada S (2015) Free silver ion as the main cause of acute and chronic toxicity of silver nanoparticles to cladocerans. Arch Environ Contam Toxicol 68(3):500-509

Sakka Y, Völkel A, Filser J (2016) Food Reduction Adds Stress to Chronic Silver Nanoparticle Toxicity for Daphnia magna. International Journal of Marine Biology and Research 1(1):1-7 Schmolke A, Bartell SM, Roy C, Green N, Galic N, Brain R (2019) Species-specific population dynamics and their link to an aquatic food web: A hybrid modeling approach. Ecol Modell 405:1-14 https://doi.org/https://doi.org/10.1016/j.ecolmodel.2019.03.024 
670 Stensberg MC, Madangopal R, Yale G, Wei Q, Ochoa-Acuña H, Wei A, Mclamore ES, Rickus

671 J, Porterfield DM, Sepúlveda MS (2014) Silver nanoparticle-specific mitotoxicity in Daphnia

672 magna. Nanotoxicology 8(8):833-842

673 Stevenson LM, Krattenmaker KE, Johnson E, Bowers AJ, Adeleye AS, McCauley E, Nisbet RM

674 (2017) Standardized toxicity testing may underestimate ecotoxicity: Environmentally relevant

675 food rations increase the toxicity of silver nanoparticles to Daphnia. Environmental toxicology

676 and chemistry 36(11):3008-3018

677 Ulm L, Krivohlavek A, Jurašin D, Ljubojević M, Šinko G, Crnković T, Žuntar I, Šikić S, Vrček

678 IV (2015) Response of biochemical biomarkers in the aquatic crustacean Daphnia magna

679 exposed to silver nanoparticles. Environmental Science and Pollution Research 22(24):19990-

68019999

681 Volker C, Boedicker C, Daubenthaler J, Oetken M, Oehlmann J (2013) Comparative toxicity

682 assessment of nanosilver on three Daphnia species in acute, chronic and multi-generation

683 experiments. PLoS One 8(10):e75026 https://doi.org/10.1371/journal.pone.0075026

684 Zhao C-M, Wang W-X (2011) Comparison of acute and chronic toxicity of silver nanoparticles

685 and silver nitrate to Daphnia magna. Environmental Toxicology and Chemistry 30(4):885-892

\section{Statements and Declarations}

\section{Funding}

690 This work was supported by the US National Science Foundation (http://www.nsf.gov/) and the

691 US Environmental Protection Agency (EPA, http://www.epa.gov/) under Cooperative

692 Agreement EF-0830117, EPA's Science to Achieve Results grant R835797, and The Worster

693 Family Research Award for undergraduate-graduate student research and mentorship at the

694 University of California, Santa Barbara.

\section{Competing Interests}

696 The authors have no relevant financial or non-financial interests to disclose.

\section{Author Contributions}

698 All authors contributed to the study conception and design. Material preparation and data

699 collection were performed by Louise M. Stevenson and Katherine E. Krattenmaker. Data

700 analysis, model development, and model analysis were performed by Louise M. Stevenson and

701 Roger M. Nisbet. The first draft of the manuscript was written by Louise M. Stevenson, and all 
702 authors commented on previous versions of the manuscript. All authors approved the final

703 manuscript.

704 Data Availability

705 The datasets generated during and/or analyzed during the current study are available from the

706 corresponding author on reasonable request. 


\section{Figures}

\section{Figure 1}

Total number of individuals in each of the daphnid populations fed different food inputs. Points are data from the daphnid population experiment: data points represent the average and the error bars their standard error. Each treatment (nanoparticle and food concentration) had 2 replicates. Note that the AgNP treatments at the highest food inputs were stopped on Day 68.

\section{Figure 2}

Fit of individual model to control Daphnia pulicaria individuals fed four food rations (Stevenson et al. 2017). Food rations are $0.0005,0.001,0.0025$ and $0.01 \mathrm{mgC} /$ daphnid/day. Negative log-likelihood value of this fit $=-2323.96$. Data points are averages and error bars represent their standard error.

\section{Figure 3}

Equilibrium biomass predictions (horizontal lines) compared to population data. Points are data from the daphnid population experiment: data points represent the average and the error bars their standard error. Each empirical treatment (nanoparticle and food concentration) had 2 replicates. The equilibrium biomass predictions represent the mean of 100 runs of the IBM per treatment (food input and AgNP concentration) and the shaded areas represent the standard deviation of these runs. We defined equilibrium as starting on Day 68 of the empirical and theoretical populations.

\section{Figure 4}

Data (points) of total number of individuals in the empirical populations compared to the IBM simulations (lines). Points are data from the daphnid population experiment: data points represent the average and the error bars their standard error. Each empirical treatment (nanoparticle and food concentration) had 2 replicates. The IBM was run 100 times per treatment and then averaged. Error bars represent standard error for the empirical data and standard deviation for the IBM simulations.

\section{Supplementary Files}

This is a list of supplementary files associated with this preprint. Click to download. 
- SupplementaryInfoforStevensonetal013022.pdf 